\title{
Unchanged EEG correlates of covert visual spatial attention after prism adaptation in healthy subjects
}

\author{
Marjolein van der Waal*a ${ }^{*}$ Inez Wijnands ${ }^{\mathrm{b}}$ \& Jason Farquhar \\ a Donders Institute for Brain, Cognition and Behaviour, Radboud University Nijmegen \\ ${ }^{\mathrm{b}}$ Radboud University Nijmegen \\ Corresponding author: Marjolein van der Waal \\ m.vanderwaal@donders.ru.nl
}

\begin{abstract}
Behavioural effects of prism adaptation in healthy subjects and neglect patients suggest a link between prism adaptation and spatial attention. A recent study found an effect of prism adaptation on several EEG correlates of spatial attention, but for two other correlates (the P3 component of the oddball ERP and alpha lateralization), the relationship remained unclear. In the current experiment, 10 healthy subjects performed a visual spatial attention task which was optimized for eliciting these two brain signals. This task was performed before and after adaptation to prism glasses with a leftward optical deviation. While prism adaptation induced a rightward bias on a pointing task, there was no effect of adaptation on behavioural performance on the spatial attention task. Moreover, the P3 component of the ERP and alpha lateralization were not influenced by prism adaptation. Together, these results show that some EEG correlates of visual spatial attention remain unchanged after prism adaptation, a finding which has its implications for current models of the neurocognitive mechanisms behind prism adaptation.
\end{abstract}

\section{Introduction}

In prism adaptation paradigms, subjects wear glasses that laterally displace eye gaze to one side while they perform a simple motor task, which usually consists of repeatedly pointing at a target in peripersonal space. After some practice, subjects adapt to the visual displacement and learn to perform the pointing task accurately. When the glasses are then removed, an aftereffect consisting of a behavioural bias towards the opposite side can be observed. Interestingly, this bias is not only present on the pointing task, but generalizes to several other tasks, many of which involve spatial attention (Jacquin-Courtois et al., 2013; Rossetti, Rode, Pisella, Farné, \& Li, 1998).

Prism adaptation not only influences performance on spatial attention tasks in healthy subjects, but also in patients with hemispatial neglect. After unilateral brain damage, patients 
with neglect fail to react to objects and events in the contralesional hemifield, despite intact motor and sensory pathways. The primary impairment in neglect seems to be a deficit in spatial attention. Adaptation to prisms with a rightward optical deviation has been shown to ameliorate a number of neglect symptoms (Newport \& Schenk, 2012; Rossetti et al., 1998) and can even be applied as a therapy for neglect (Kerkhoff \& Schenk, 2012; Newport \& Schenk, 2012).

Until now, the effects of prism adaptation have primarily been studied on the behavioural level. However, spatial attention is reflected in a number of neurophysiological signals, for example the amplitude of several event-related potential (ERP) components, including N1, P1 and P3. These components are larger in response to stimuli occurring at attended as compared to unattended locations (Coull, 1998; Polich, 2007). Additionally, oscillations in the alpha band (8-14 Hz) from posterior brain areas show a particular topography depending on the direction of visual spatial attention: when attention is directed to one half of the visual field, an increase in alpha power is observed over ipsilateral posterior areas, while alpha power decreases over contralateral posterior areas (Worden, Foxe, Wang, \& Simpson, 2000).

A recent study found neglect-like changes in the N1 and P1 components of the ERP after adaptation to left-deviating prisms in healthy subjects, suggesting that similar mechanisms may be involved in neglect symptoms and in prism adaptation (Martín-Arévalo et al., 2016). However, this study did not find an effect of prism adaptation on P3 in healthy subjects, whereas changes in the P3 component have been reported in neglect patients (Saevarsson, Kristjánsson, Bach, \& Heinrich, 2012). One reason for this discrepancy could be that the P3 is best observed in an oddball paradigm, which was employed in the study with patients (Saevarsson et al., 2012) but not in the study with healthy subjects (Martín-Arévalo et al., 2016). There has been no research on alpha lateralization in neglect patients or after prism adaptation in healthy subjects so far. However, the results of the previous EEG study with healthy subjects support the idea that prism adaptation influences attention by acting on the dorsal attention network (Martín-Arévalo et al., 2016). It has also been shown that the dorsal attention network is causally involved in regulating alpha lateralization in the visual cortex (Capotosto, Babiloni, Romani, \& Corbetta, 2009; Liu, Bengson, Huang, Mangun, \& Ding, 2016). Therefore, if prism adaptation alters activity in the dorsal attention network, it is to be expected that this will lead to changes in alpha lateralization as well.

In this experiment, we investigate the effect of prism adaptation on two electrophysiological correlates of spatial attention, the P3 and alpha lateralization, in healthy subjects. If prism adaptation induces changes in spatial attention-related brain signals in healthy subjects that are similar to the brain signals of neglect patients, we expect that the P3 amplitude in response to left-sided stimuli will decrease after adaptation, and that the pattern of alpha lateralization will change after prism adaptation. In addition to studying the grand average attention-related brain signals, we will use machine learning techniques to predict the direction of spatial attention on the single trial level, using the decoding accuracy as a measure of the similarity of single brain responses before and after adaptation. 


\section{Methods}

\section{Participants}

Ten right-handed healthy subjects (7 male), aged 19-26 (mean 21) years, participated in this experiment. Subjects did not have any neurological abnormalities, reported normal or corrected to normal vision, and did not use medication. The experiment was approved by the ethical committee of the Faculty of Social Sciences of the Radboud University and all participants gave written informed consent prior to the experiment.

\section{Materials}

EEG was recorded with 64 sintered $\mathrm{Ag} / \mathrm{AgCl}$ active electrodes (BioSemi, Amsterdam, The Netherlands), placed according to the international 10-20 system, at a sampling rate of $2048 \mathrm{~Hz}$. Simultaneously, eye gaze was recorded with an EyeLink 1000 eyetracker (SR Research Ltd., Ontario, Canada) at a sampling rate of $1000 \mathrm{~Hz}$. The subject's head was stabilized on a chin rest. Visual stimuli were presented on a 17" TFT screen with $800 \times 600$ pixel resolution and a refresh rate of $60 \mathrm{~Hz}$.

Subjects were seated in front of a table. The screen and the eyetracking camera were placed in the middle of the table at a distance of approximately $60 \mathrm{~cm}$ from the subject. A button box was placed directly in front of the subject.

\section{Stimuli}

A schematic representation of the stimuli is shown in Figure $1 \mathrm{~b}$. A fixation cross of $1 \times 1 \mathrm{~cm}$ $\left(1.0^{\circ} \times 1.0^{\circ}\right)$ was presented in the middle of the screen, with two white squares $5.5 \mathrm{~cm}\left(5.2^{\circ}\right)$ to the side and $2.2 \mathrm{~cm}\left(2.0^{\circ}\right)$ below the fixation cross. The squares were $4.2 \times 4.2 \mathrm{~cm}\left(4.0^{\circ} \times 4.0^{\circ}\right)$ in size.

On top of the white squares, blue letters were presented, one at a time. These letters were $1 \times 1$ $\mathrm{cm}\left(1.0^{\circ} \times 1.0^{\circ}\right)$ in size and located in the centre of the white squares. Letters were presented sequentially for 0.2 seconds, with a stimulus onset asynchrony (SOA) of 0.3 seconds. 


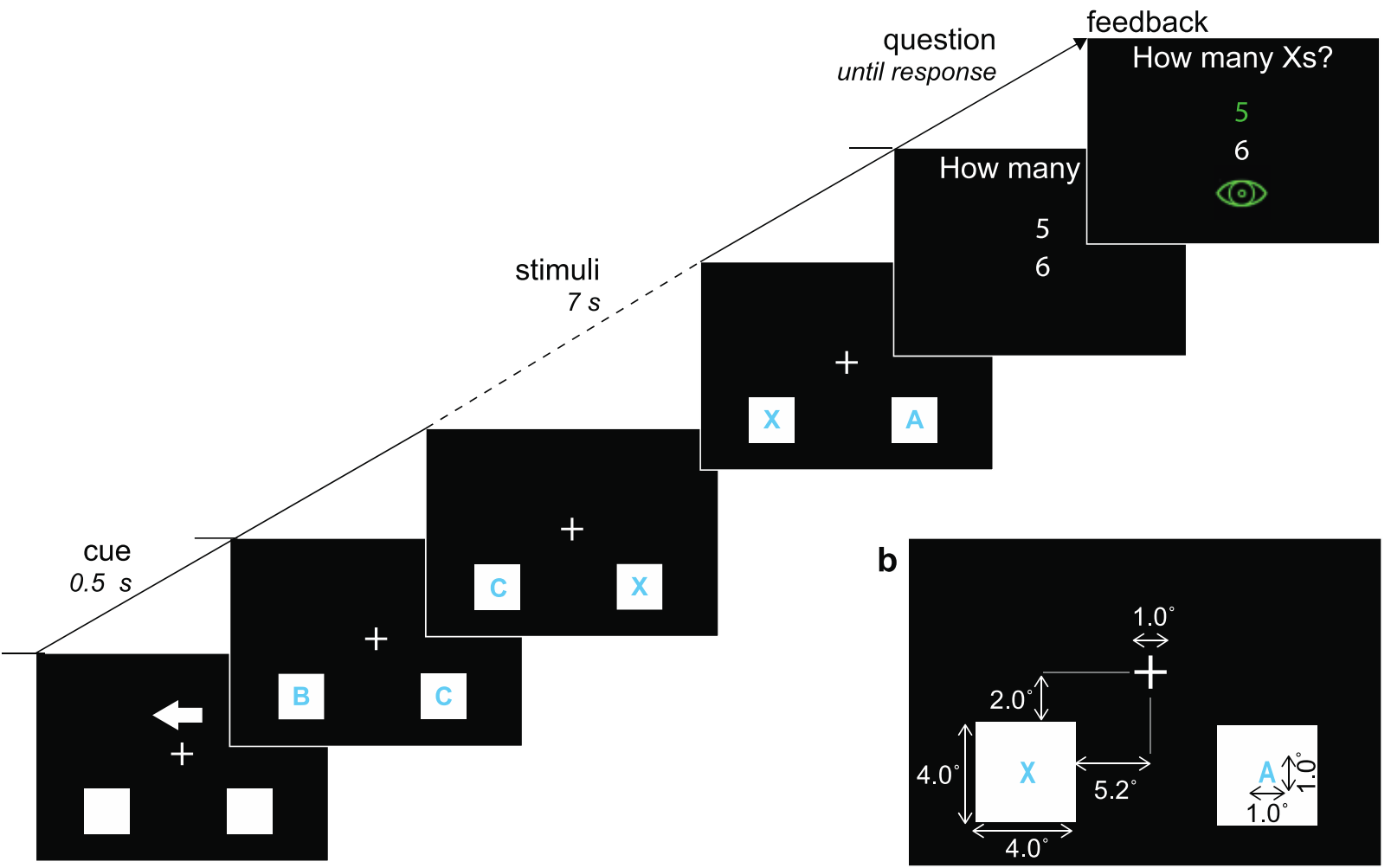

Figure 1: Schematic overview of the experimental design. a) Trial timeline. Each trial started with an arrow cue indicating which side should be attended. Subsequently, a random sequence of 22 letters (A,B,C, and X) was presented on top of each square, with an SOA of 0.3 seconds. At the end of the trial subjects were asked to report how many times they had counted the letter $X$ on the indicated side and they received feedback about their answer and eye gaze. b) Stimulus display. Size and positioning of the stimuli on the computer screen.

\section{Task}

The task was designed to allow for simultaneous measurement of stimulus-locked ERPs and alpha lateralization (see (Van der Waal, Farquhar, Fasotti, \& Desain, 2017) for a similar task). A schematic overview of a trial is shown in Figure 1a. Each trial started with the fixation cross and an arrow pointing either to the left or to the right square for 0.5 seconds. Then a sequence containing the letters $A, B, C$ and $X$ was presented in pseudorandom order on top of the white squares. The letter sequences on the left and right were independent from each other and each sequence could contain either five or six times the letter $X$, with equal probability. The subjects' task was to count the number of times they saw the letter $X$ on the side indicated by the arrow. There was always at least one other letter between two X's and X's never appeared on the left and on the right simultaneously. Each letter sequence lasted 7 seconds.

At the end of the trial, subjects were asked to indicate with a button press whether they counted 5 or $6 \mathrm{X}^{\prime} \mathrm{s}$. While subjects performed the task, their eye gaze was monitored online. At the end of the trial, subjects received points for a correct answer and for maintaining eye gaze on the fixation cross throughout the trial.

Participants first completed a practice block, followed by one block of 60 trials. After they had adapted to the prism glasses, they completed another three blocks of 20 trials, with the adaptation procedure repeated between blocks. 


\section{Prism adaptation}

During the adaptation phase, subjects wore prism glasses with a leftward optical deviation of $15^{\circ}$. In front of the participant, a piece of paper (A4 format, landscape orientation) was taped to the desk with a dot in the middle and two star-shaped figures at the left and right border. The participants were asked to point at the stars with their right hand, in a steady pace and randomly alternating left and right movements. The first adaptation phase lasted 15 minutes, the next two re-adaptations between experimental blocks lasted 10 minutes.

Immediately after the glasses were removed, participants were asked to close their eyes, point at the dot in the middle of the sheet of paper and keep their finger on the paper until they were told to remove their hand. The deviation from the dot was measured in centimetres (positive number for the right side and negative for the left side) and was not reported to the participant. During experimental blocks, participants were asked to use their right hand as little as possible and keep their eyes closed between trials. Subjects were asked to point at the dot with closed eyes again after each experimental block, to determine if an aftereffect of prism adaptation was still present.

\section{Eye gaze analysis}

The position of subjects' gaze was analysed after each trial, making use of the EyeLink online parsing system, which parses the data into saccade, blink and fixation events. These events were streamed to our software for analysis. Trials which contained a saccade or blink event, and trials where the average gaze position deviated more than $3^{\circ}$ from the fixation cross in any direction, were excluded from further analysis.

\section{EEG preprocessing}

The EEG was analysed using the same methods as (Van der Waal et al., 2017). First, the raw EEG data was sliced into epochs from 0.5-6.5 seconds after cue offset. This window contained 20 ERP epochs, 5 of which were target epochs. For ERP and alpha lateralization analysis, the EEG contains no relevant information in frequencies above $100 \mathrm{~Hz}$, so for computational reasons the data was downsampled to $256 \mathrm{~Hz}$. The data was re-referenced to the mean of all electrodes. A channel was marked as bad if the power in this channel over all trials was more than 3.5 times the standard deviation of the average channel power. Similarly, a trial was marked as bad if the power in this trial over all channels was more than 3.5 times the standard deviation of the average trial power. Bad trials and channels were removed, and a new common average reference was performed to compensate for the removed trials and channels. Rejected channels were interpolated using spherical spline interpolation (Perrin, Pernier, Bertrand, \& Echallier, 1989). From that point, there were separate analysis pipelines for analysing ERPs and alpha lateralization.

For ERP analysis, the sliced data was linearly detrended and an FFT bandpass filter for frequencies of $0.5-12 \mathrm{~Hz}$ was applied (Farquhar \& Hill, 2013). The data was further downsampled to $32 \mathrm{~Hz}$ to reduce computational load and subsliced into epochs from 0-600 ms after the presentation of each stimulus (letter), excluding the first and last stimulus of a sequence. The small epochs were linearly detrended.

Alpha lateralization was analysed by computing a spherical spline current source density (CSD) 
transform (which is equivalent to a scalp laplacian) of the data from 0.5-6.5 seconds after stimulus onset, using the CSD toolbox of Kayser \& Tenke (2006). The data was subsliced into windows of 2 seconds and for each window the power spectrum was computed using the Welch method. The result was normalized per subject such that each frequency's average power over all trials was 1 and log transformed.

\section{Single trial classification}

The traditional approach to study spatial attention-related brain signals is to collect a large number of trials and compute an average brain response over all subjects and trials. While averaging over many trials has the benefit of improving the signal to noise ratio, subtle changes in the brain responses between trials are lost. To overcome this problem, the grand average approach can be complemented by single trial analysis using machine learning techniques. The accuracy with which the direction of attention can be decoded on the single trial level is a measure of the consistency of the brain signals on the single trial level: the more consistent the brain response in every single trial, the more reliably the direction of attention can be decoded. Classifiers were trained on the data of the condition before prism adaptation and tested on the data of both conditions.

Following the analysis procedure of (Farquhar \& Hill, 2013), responses to transient stimuli were spatially whitened to remove cross correlation between channels. Only responses to letters $X$ on the attended side (targets) and unattended side (non-targets) were used as input for the classifier and a regularized linear logistic regression classifier (rLLR) was trained to separate the two classes using ten-fold cross validation to set the $L 2$ regularization strength. Classification performance was calculated for this binary problem, resulting in 20 decision values per trial (one for every stimulus). Thus, in this classification procedure we separated responses to target letters from responses to non-target letters, however we are interested in separating attention directed to the left from attention directed to the right. To determine whether attention was directed to the left or to the right, we combined the 20 decision values within each trial as follows. Every trial has a codebook, which is a matrix of 2 sides (left and right) by 20 stimuli that indicates for each side- stimulus-combination whether it is a target stimulus (letter $\mathrm{X}$ ) or not. At the end of the trial the 20 decision values for the target versus non-target classification were multiplied by the codebook and summed, resulting in a decision value for right-sided attention and one for left-sided attention. The side with the highest decision value was selected. For classifying alpha lateralization, we used the power in the frequencies from $8-14 \mathrm{~Hz}$ at occipital electrodes (PO3, PO4, P07, PO8, POz, 01, 02, Oz). Again an rLLR classifier was trained with ten-fold cross validation and classification performance was calculated for the binary problem.

\section{Statistical analyses}

For all statistical tests, the significance level was set to 0.05 . When multiple comparisons were made, the alpha level of pairwise comparisons was Bonferroni corrected for the number of comparisons. For ANOVAs the SPSS Statistics 21.0 software package was used, while the clusterbased permutation tests were performed with FieldTrip (Oostenveld, Fries, Maris, \& Schoffelen, 2011). 
Prism adaptation: For investigating the effect of prism adaptation on pointing at a mark in front of the body midline, we performed a $2 \times 3$ repeated measures ANOVA on the difference between the pointing deviation at baseline and at each test moment. This ANOVA had two factors: test moment (beginning or end of block) and block number (first, second or third).

Behavioural performance: For comparing behavioural performance before and after adaptation, we performed a $2 \times 2$ repeated measures ANOVA on behavioural accuracies with condition (before or after adaptation) and attended side (left or right) as factors.

Electrophysiological responses before and after adaptation: We compared the attention-related ERPs and alpha lateralization before and after prism adaptation. For ERP responses to transient stimuli, we computed a target versus non-target difference waveform for each condition and stimulus side. The difference waves of the two conditions were compared using a cluster-based permutation test (Maris \& Oostenveld, 2007). Since a separate clustertest was performed for left and right-sided stimuli, the alpha level for these tests was set to 0.025 .

Alpha modulation was calculated by subtracting the power spectrum of trials with rightwards attention from the power spectrum of trials with leftwards attention and dividing the difference by the sum of the two power spectra. We then compared the alpha modulation spectra before and after adaptation using a cluster-based permutation test on the frequencies from 8 to $14 \mathrm{~Hz}$.

Single trial classification before and after adaptation: The final objective of this study was to compare classification performance before and after prism adaptation. To this end, we performed a $2 \times 2 \times 2$ repeated measures ANOVA on the classification accuracies with condition (before or after adaptation), brain response (ERPs or alpha lateralization) and attended side (left or right) as factors.

\section{Results}

\section{Number of trials}

On average, 49 trials (containing 980 ERP epochs) remained in each condition after artefact rejection and rejection of trials containing blinks or eye movements. The number of trials determines the confidence interval around the chance level of the classification procedure (Müller-Putz, Scherer, Brunner, Leeb, \& Pfurtscheller, 2008). Given the number of trials in this experiment, classification accuracies of 0.63 and higher are considered significantly better than chance level.

\section{Prism adaptation}

Figure 2 shows the deviation in centimetres when subjects pointed at a mark in front of their body midline with closed eyes. The baseline measure was not taken on the same day as the experiment, but several weeks later, due to an oversight. Prism adaptation was not assessed for one subject, so these results are based on nine subjects. The effect of adaptation on pointing accuracy was stronger at the beginning than at the end of experimental blocks $\left(F_{1,8}=14.5, p=\right.$ $\left.0.009, \eta_{p}{ }^{2}=0.71\right)$. However, three subsequent repeated measures t-tests showed that the 
pointing deviation at the end of each block was still significantly larger than baseline (block 1: mean difference $=4.7, \mathrm{t}_{7}=6.3, \mathrm{p}<0.001$; block 2 : mean difference $=6.1, \mathrm{t}_{8}=11.9, \mathrm{p}<0.001$; block 3: mean difference $=6.1, t_{7}=19.2, p<0.001$ ).

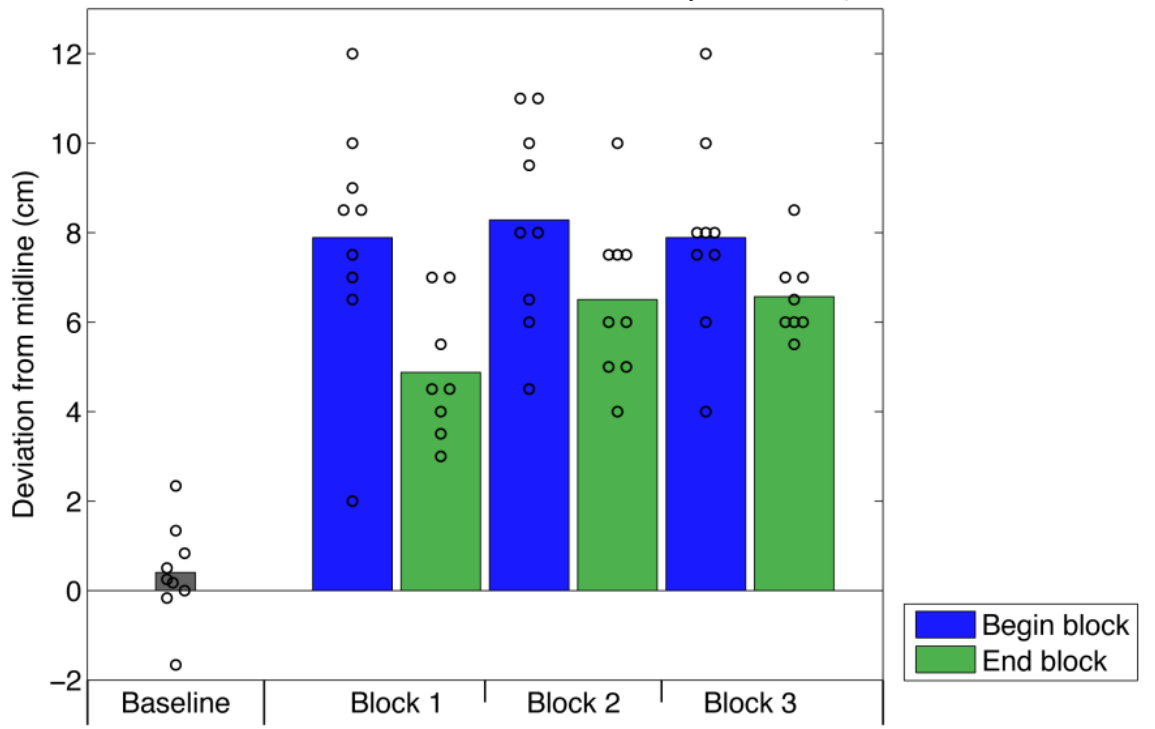

Fig 2 Effect of prism adaptation on pointing accuracy. Bars show the deviation from the midline target in centimeters at the start and end of each experimental block. The circles show values of individual subjects. Positive numbers reflect rightward deviations

\section{Behavioural performance}

The behavioural performance on the counting task is shown in Figure 3. On average, subjects counted the correct number of $X^{\prime}$ 's in about $70 \%$ of the trials. There was no significant effect of adaptation or stimulus side on behavioural performance.

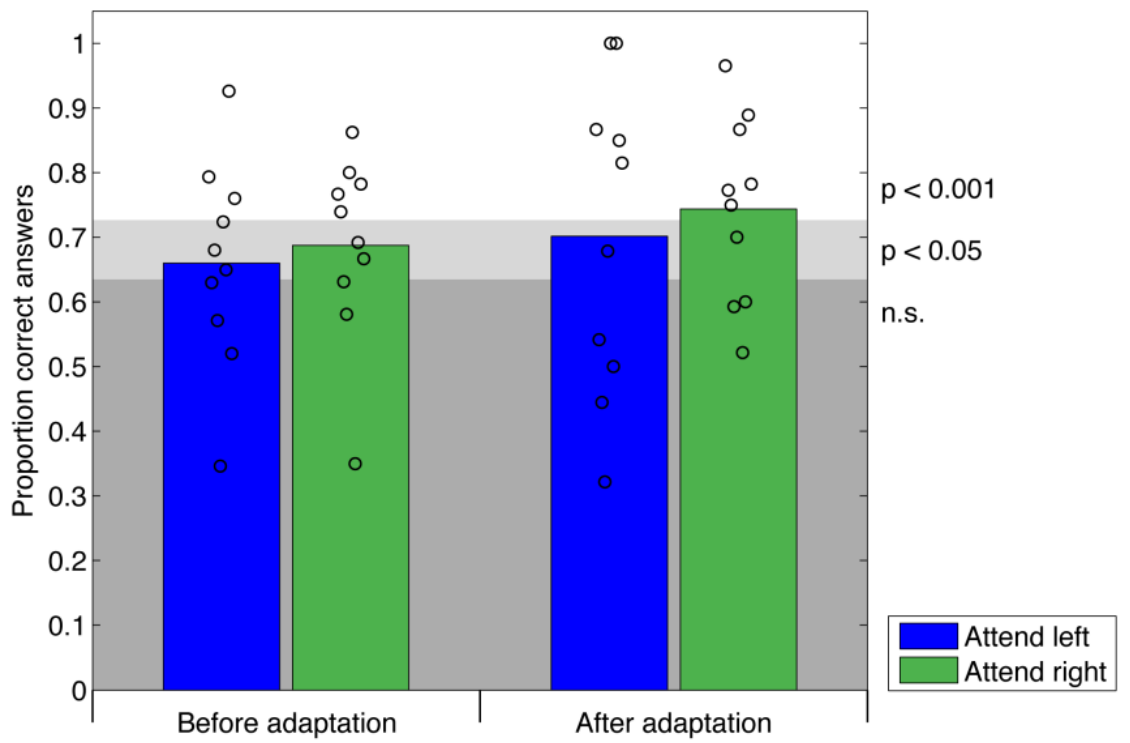

Fig 3 Behavioural performance on the task. Bars show the average proportion of trials in which the correct number of letters X was reported. Circles show the accuracies of individual subjects. Grey and white areas indicate whether performance is significantly better than chance level 


\section{Electrophysiology}

\section{ERPs}

The grand average responses to target (letter $X$ on the attended side) and non-target (all other letters) stimuli at electrode $\mathrm{CPz}$ are shown in Figure 4. A cluster-based permutation test showed no significant differences between the average target - non-target difference before and after adaptation for either left or right stimuli.

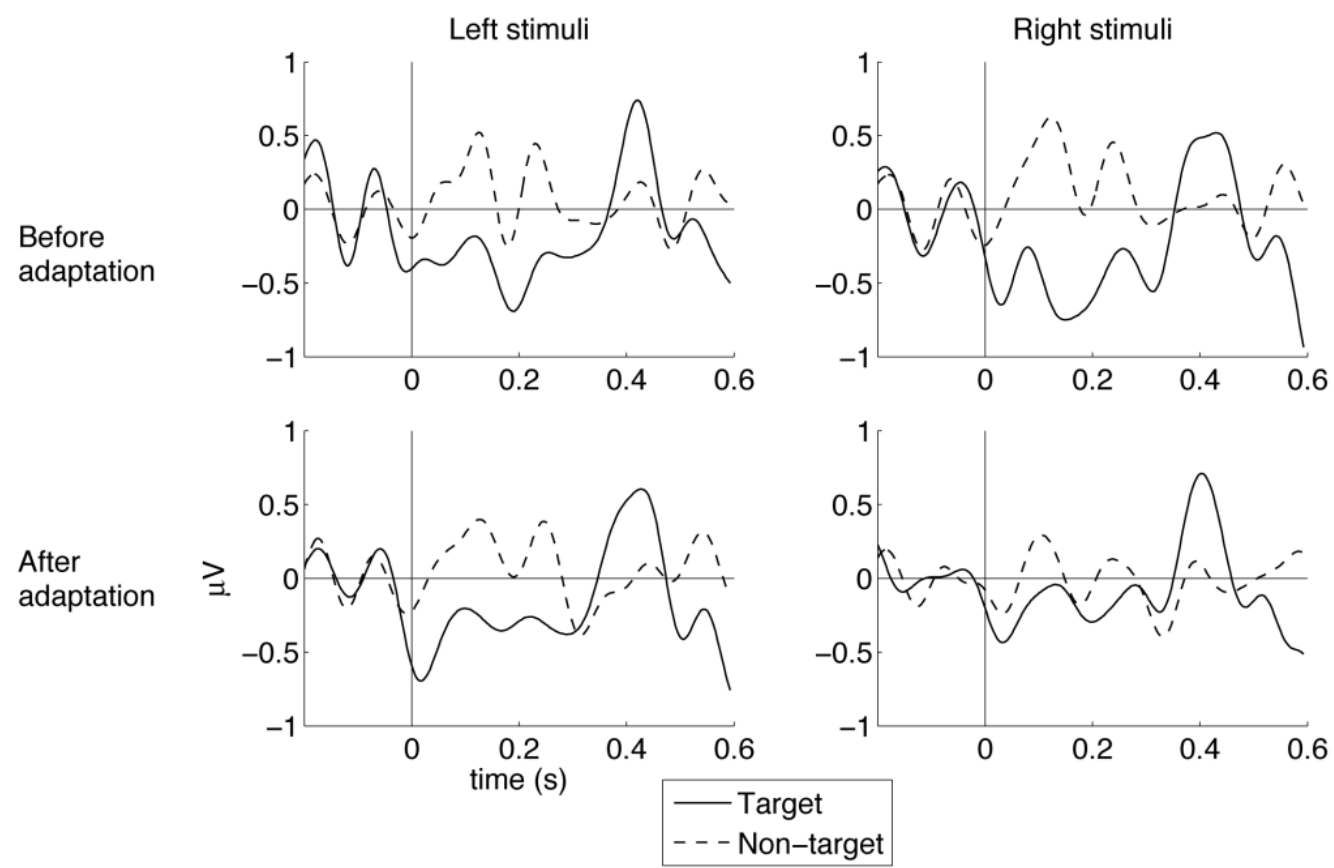

Fig 4 Grand average ERPs. Average brain response to target stimuli (solid line) and non-target stimuli (dashed line) at electrode $\mathrm{CPz}$

\section{Alpha modulation}

Alpha modulation before and after prism adaptation is shown in Figure 5. Alpha modulation is calculated by subtracting the average alpha power when attending to the left visual field from the average alpha power when attending to the right visual field and dividing the difference by the sum of both (Roijendijk, Farquhar, van Gerven, Jensen, \& Gielen, 2013). The pattern of alpha power modulation before and after adaptation was compared using a cluster-based permutation test, which revealed no significant differences between the two conditions.
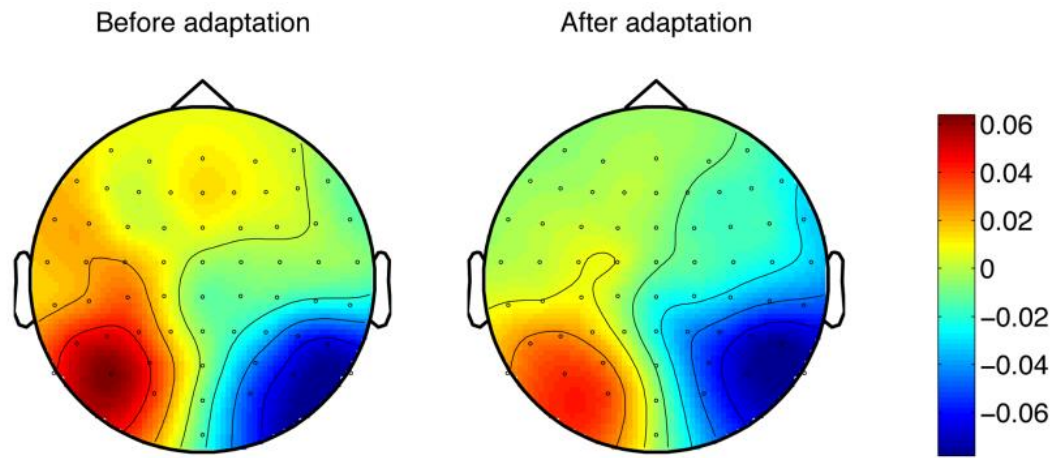

Fig 5 Alpha modulation. Modulation of power in the alpha band (8-14 Hz), before and after prism adaptation 


\section{Classification}

The direction of attention (left or right) could be decoded from single trial ERPs or lateralized alpha with above chance level accuracy in both conditions (see Figure 6). There was no effect of adaptation, brain response (ERPs or alpha modulation) or attended side on classification accuracy. In other words, adaptation to prisms did not induce a significant change in single trial brain responses.

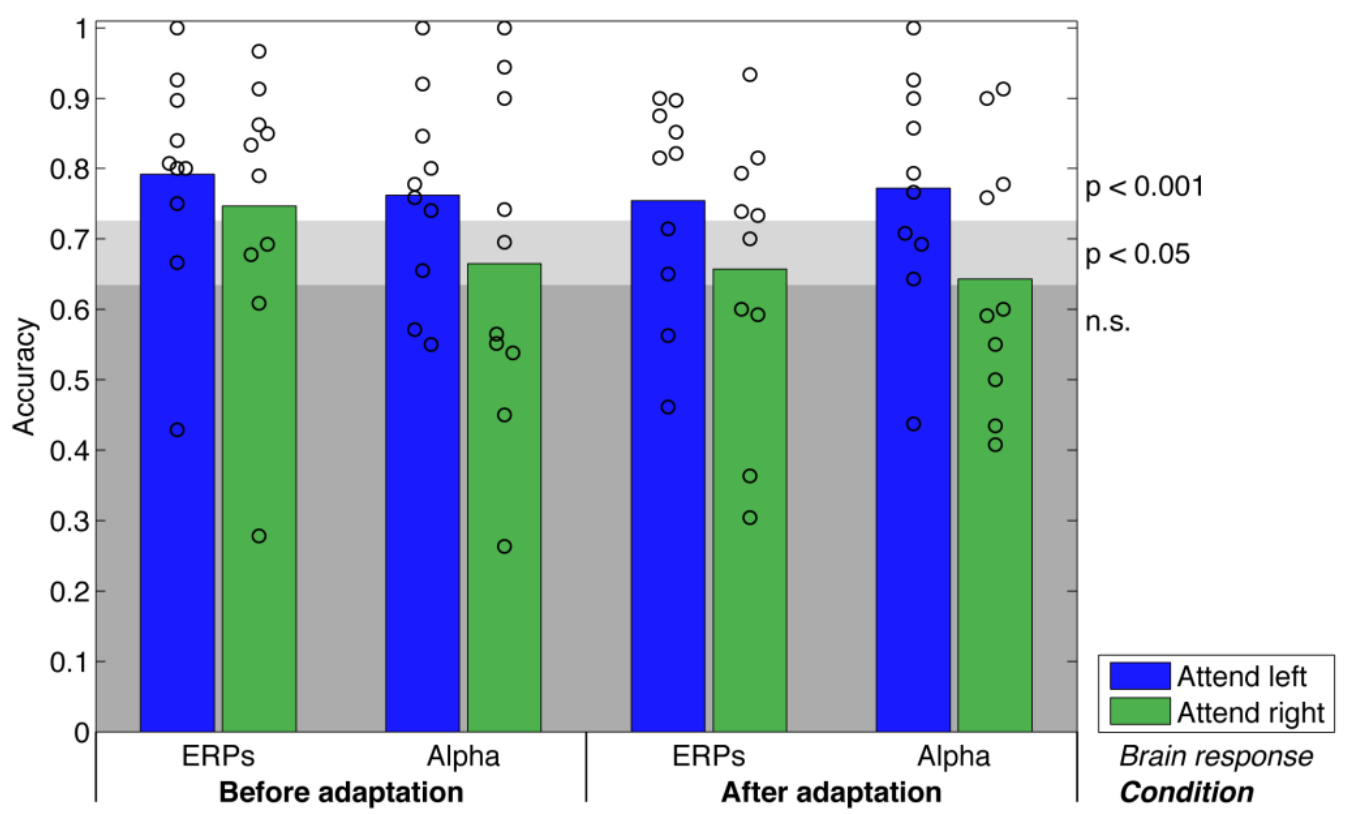

Fig 6 Decoding accuracies. Bars show the proportion of trials in which the direction of attention could be correctly decoded from ERPs or lateralized alpha. Circles show the decoding accuracies for individual subjects. Grey and white areas indicate whether decoding accuracy is significantly better than chance level

\section{Statistical power}

In order to judge how confident we can be about the absence of an effect of prism adaptation on behaviour and electrophysiological signals, we estimated the power of our statistical tests. The power of a test depends on the number of subjects, the error probability alpha, and the effect size. While the first two parameters are known, the effect size has to be estimated based on the literature. We computed the Cohen's d effect size of prism adaptation on behavioural outcome measures in healthy subjects from previous studies that reported the mean and standard deviation or standard error of their detected difference (Bultitude, Van der Stigchel, \& Nijboer, 2013; Colent, Pisella, Bernieri, Rode, \& Rossetti, 2000; Michel et al., 2003; Reed \& Dassonville, 2014). These effect sizes ranged from 0.18 to 1.4 , with an average of 0.75 standard deviations. The studies included 11 participants on average. With our sample number of 10 subjects and critical alpha level of 0.05 , there was a 0.56 probability of detecting an effect of prism adaptation on task performance the size of 0.75 standard deviations. Effect sizes of 1 standard deviation and larger would have been detected with a probability of more than 0.8 . These power values were computed with the G*power program (Faul, Erdfelder, Lang, \& Buchner, 2007).

For the cluster-based permutation test on ERPs, we estimated the effect sizes we were able to detect using a simulation method. We decreased the amplitude of the target versus non-target 
difference wave after adaptation of each individual subject at the channels and time points where the P3 component was present. We chose to simulate a change in the P3 of trials with left-sided attention because that is the effect we expected to find. The ERP amplitudes were gradually decreased in steps of $10 \%$ and after each decrease the artificially reduced signal was tested against the difference wave before adaptation using the cluster-based permutation test. The cluster-based permutation test was able to detect a P3 amplitude reduction of $50 \%$ or more.

\section{Discussion}

In this experiment, we studied the effect of prism adaptation on spatial attention-related brain responses in healthy subjects. Although the prism adaptation procedure successfully induced a rightward bias on the pointing task, it did not lead to a change in performance on the visual spatial attention task or a change in the P3 component of the oddball ERP or a change in alpha lateralization.

It is always harder to be certain of the absence of an effect than of its presence. Although a negative result can never be proven, we estimated the likelihood that we would have found effects of different sizes, had they been there. Overall, we had a good chance of finding effects of a similar size as previous prism adaptation studies. Therefore, we believe that the negative result of this study is meaningful.

Our first finding, the absence of an effect of prism adaptation on behavioural task performance, is in contrast to the results of another recent study which did find prism adaptation-induced changes in performance on a covert visual attention task in healthy subjects(Martín-Arévalo et al., 2016). Several differences between the two covert attention tasks may explain this discrepancy. The first difference is the duration of the period in which spatial attention had to be employed. Whereas the previous study used a Posner-like task with a cue-target interval of 300-400 ms, our task required subjects to sustain spatial attention for about 7 seconds per trial. Possibly, prism adaptation primarily affects covert spatial attention when it has to be employed on a short timescale. Secondly, in the previous study, one stimulus appeared on the left or on the right, whereas in our study, stimuli appeared on the left and right side simultaneously. It could be that prism adaptation influences the perception of single stimuli more than competing stimuli. Finally, eye movements were monitored more strictly in the present study. As prism adaptation influences eye movements (Angeli, Benassi, \& Làdavas, 2004; Bultitude et al., 2013), the effects of the previous study may partly be mediated by altered gaze behaviour.

Although task differences might explain the differential effects found in healthy subjects, the absence of a behavioural effect in the present study is also in contrast to the results in neglect patients. In neglect patients, a significant correlation was found between neglect severity and ability to detect oddball stimuli on the contralesional side (Saevarsson et al., 2012). Although there are many studies showing neglect-like behaviour in healthy subjects after prism adaptation, there are also examples where prism adaptation did not induce certain neglect-like symptoms in healthy subjects(Jacquin-Courtois et al., 2013). For instance, one study found an effect of prism adaptation on temporal order judgements in neglect patients, but not in healthy subjects (Berberovic, Pisella, Morris, \& Mattingley, 2004). In addition, two treatment methods 
for neglect, neck-proprioceptive and caloric-vestibular stimulation, which induce changes in covert attention in neglect patients, have failed to induce similar changes in healthy subjects (Rorden, Karnath, \& Driver, 2001).

Our second finding, the absence of an effect of prism adaptation on the P3 component of the ERP is in line with the findings of (Martín-Arévalo et al., 2016), even though we used a task which was optimized for studying the P3 component and which was similar to the task that did demonstrate abnormalities in the P3 component in neglect patients (Saevarsson et al., 2012). Thus, prism adaptation in healthy subjects fails to induce a change in behaviour as well as an EEG correlate of visual spatial attention that is present in patients with neglect. Clearly, prism adaptation in healthy subjects can model some, but not all, aspects of the neglect syndrome. A better insight into how prism adaptation works on the neurophysiological level might help us understand the differences between its effects in the healthy brain versus its effects in the brains of patients with neglect.

Neuroimaging studies show that the cerebellum and parietal cortex are involved in the adaptation phase of the prism adaptation procedure (Newport \& Schenk, 2012). However, not much is known about the neural changes during the aftereffect of prism adaptation. Given the changes in spatial attention behaviour after prism adaptation, one might expect changes in the brain networks associated with spatial attention. In the attention network theory of Corbetta and Shulman (Corbetta \& Shulman, 2002), a dorsal and ventral attention network are identified. The goal-driven dorsal attention network exerts top-down control over sensory areas to bias the processing of incoming stimuli. Behavioural studies using Posner tasks indicate that top-down attention processes are altered after prism adaptation (Nijboer, Mcintosh, Nys, Dijkerman, \& Milner, 2008; Striemer, Sablatnig, \& Danckert, 2006). Neuroimaging studies show that activity in the dorsal attention network is altered in patients with neglect (Corbetta, Kincade, Lewis, Snyder, \& Sapir, 2005) and changes after prism adaptation (Saj, Cojan, Vocat, Luauté, \& Vuilleumier, 2013). The authors of the recent EEG study (Martín-Arévalo et al., 2016) also interpreted their findings as supporting the theory that prism adaptation exerts its influence on spatial attention mainly via the dorsal attention network. However, the results of our study do not support the idea that prism adaptation changes dorsal network activity, because it has been shown that the dorsal attention network controls visual spatial attention through alpha lateralization in the visual cortex (Capotosto et al., 2009; Liu et al., 2016). Therefore, the fact that we did not observe changes in alpha lateralization after prism adaptation suggests that dorsal attention network activity during this task was not affected by prism adaptation.

The second network in the attention network theory is the ventral attention network, which is responsible for stimulus-driven attention and is important for the reorienting of attention (Corbetta \& Shulman, 2002). On the behavioural level, the effects of prism adaptation on stimulus-driven attention and reorienting are mixed, both for neglect patients (Nijboer et al., 2008; Schindler et al., 2009; Striemer \& Danckert, 2007) and healthy subjects (Schindler et al., 2009; Striemer et al., 2006). On the neurophysiological level, activity in the ventral attention network is changed in patients with neglect (Corbetta et al., 2005) and a recent fMRI study also found evidence for altered neural processing in the ventral attention network after prism adaptation in healthy subjects (Crottaz-Herbette, Fornari, \& Clarke, 2014). This finding seems to be in contrast to our results, because the P3 component of the ERP, which has been reliably 
associated with the ventral attention network (Corbetta, Patel, \& Shulman, 2008), was unchanged after prism adaptation.

In conclusion, the present study failed to show an effect of prism adaptation on visual spatial attention in healthy subjects, on the behavioural as well as the electrophysiological level. This result was unexpected given recent behavioural and neurophysiological studies on the effects of prism adaptation. One explanation for the discrepancy between our study and previous ones may be in the task that was used, emphasizing that the effects of prism adaptation in the healthy brain are strongly task-dependent. However, the question then remains why a task that successfully showed altered behaviour and electrophysiological signals in patients with neglect, failed to induce similar effects in healthy subjects. Identification of the specific elements of attention which are affected by prism adaptation may help us to better understand the neurocognitive mechanisms behind prism adaptation in the healthy brain and in patients with neglect.

\section{References}

Angeli, V., Benassi, M. G., \& Làdavas, E. (2004). Recovery of oculo-motor bias in neglect patients after prism adaptation. Neuropsychologia, 42, 1223-1234.

http://doi.org/10.1016/j.neuropsychologia.2004.01.007

Berberovic, N., Pisella, L., Morris, A. P., \& Mattingley, J. B. (2004). Prismatic adaptation reduces biased temporal order judgements in spatial neglect. Neuroreport, 15(7), 1199-1204.

Bultitude, J. H., Van der Stigchel, S., \& Nijboer, T. C. W. (2013). Prism adaptation alters spatial remapping in healthy individuals: Evidence from double-step saccades. Cortex, 49(3), 759-770.

Capotosto, P., Babiloni, C., Romani, G. L., \& Corbetta, M. (2009). Frontoparietal Cortex Controls Spatial Attention through Modulation of Anticipatory Alpha Rhythms. Journal of Neuroscience, 29(18), 5863-5872. http://doi.org/10.1523/JNEUROSCI.0539-09.2009

Colent, C., Pisella, L., Bernieri, C., Rode, G., \& Rossetti, Y. (2000). Cognitive bias induced by visuo-motor adaptation to prisms: a simulation of unilateral neglect in normal individuals? Neuroreport, 11(9), 1899-1902.

Corbetta, M., Kincade, M. J., Lewis, C., Snyder, A. Z., \& Sapir, A. (2005). Neural basis and recovery of spatial attention deficits in spatial neglect. Nature Neuroscience, 8(11), 1603-10. http://doi.org/10.1038/nn1574

Corbetta, M., Patel, G., \& Shulman, G. L. (2008). The Reorienting System of the Human Brain: From Environment to Theory of Mind. Neuron, 58(3), 306-324.

Corbetta, M., \& Shulman, G. L. (2002). Control of Goal-Directed and Stimulus-Driven Attention in the Brain. Nature Reviews Neuroscience, 3(3), 215-229. http://doi.org/10.1038/nrn755

Coull, J. T. (1998). Neural correlates of attention and arousal: insights from electrophysiology, functional neuroimaging and psychopharmacology. Progress in Neurobiology, 55(4), 343-361.

Crottaz-Herbette, S., Fornari, E., \& Clarke, S. (2014). Prismatic Adaptation Changes Visuospatial Representation in the Inferior Parietal Lobule. Journal of Neuroscience, 34(35), 11803-11811. http://doi.org/10.1523/JNEUROSCI.3184-13.2014

Farquhar, J., \& Hill, N. J. (2013). Interactions between pre-processing and classification methods for event-related-potential classification: best-practice guidelines for brain-computer interfacing. Neuroinformatics, 11(2), 175-192.

Faul, F., Erdfelder, E., Lang, A.-G., \& Buchner, A. (2007). G*Power 3: a flexible statistical power analysis program for the social, behavioral, and biomedical sciences. Behavior Research Methods, 39(2), 
175-191. http://doi.org/10.3758/BF03193146

Jacquin-Courtois, S., O’Shea, J., Luauté, J., Pisella, L., Revol, P., Mizuno, K., ... Rossetti, Y. (2013).

Rehabilitation of spatial neglect by prism adaptation. A peculiar expansion of sensorimotor after-

effects to spatial cognition. Neuroscience and Biobehavioral Reviews, 37(4), 594-609.

http://doi.org/10.1016/j.neubiorev.2013.02.007

Kayser, J., \& Tenke, C. E. (2006). Principal components analysis of Laplacian waveforms as a generic method for identifying ERP generator patterns: II. Adequacy of low-density estimates. Clinical Neurophysiology, 117(2), 369-380.

Kerkhoff, G., \& Schenk, T. (2012). Rehabilitation of neglect: An update. Neuropsychologia, 50(6), 10721079. http://doi.org/10.1016/j.neuropsychologia.2012.01.024

Liu, Y., Bengson, J., Huang, H., Mangun, G. R., \& Ding, M. (2016). Top-down Modulation of Neural Activity in Anticipatory Visual Attention: Control Mechanisms Revealed by Simultaneous EEG-fMRI. Cerebral Cortex, 26, 517-529. http://doi.org/10.1093/cercor/bhu204

Maris, E., \& Oostenveld, R. (2007). Nonparametric statistical testing of EEG- and MEG-data. Journal of Neuroscience Methods, 164(1), 177-190.

Martín-Arévalo, E., Laube, I., Koun, E., Farne, A., Reilly, K. T., \& Pisella, L. (2016). Prism Adaptation Alters Electrophysiological Markers of Attentional Processes in the Healthy Brain. Journal of Neuroscience, 36(3), 1019-1030. http://doi.org/10.1523/JNEUROSCI.1153-15.2016

Michel, C., Pisella, L., Halligan, P. W., Luauté, J., Rode, G., Boisson, D., \& Rossetti, Y. (2003). Simulating unilateral neglect in normals using prism adaptation: implications for theory. Neuropsychologia, 41(1), 25-39.

Müller-Putz, G. R., Scherer, R., Brunner, C., Leeb, R., \& Pfurtscheller, G. (2008). Better than random? A closer look on $\mathrm{BCl}$ results. International Journal of Bioelectromagnetism, 10(1), 52-55.

Newport, R., \& Schenk, T. (2012). Prisms and neglect : What have we learned? Neuropsychologia, 50(6), 1080-1091. http://doi.org/10.1016/j.neuropsychologia.2012.01.023

Nijboer, T. C. W., Mcintosh, R. D., Nys, G. M. S., Dijkerman, H. C., \& Milner, A. D. (2008). Prism adaptation improves voluntary but not automatic orienting in neglect. NeuroReport, 19(3), 293-298.

Oostenveld, R., Fries, P., Maris, E., \& Schoffelen, J.-M. (2011). FieldTrip: Open source software for advanced analysis of MEG, EEG, and invasive electrophysiological data. Computational Intelligence and Neuroscience, 2011, 156869.

Perrin, F., Pernier, J., Bertrand, O., \& Echallier, J. F. (1989). Spherical splines for scalp potential and current density mapping. Electroencephalography and Clinical Neurophysiology, 72(2), 184-187.

Polich, J. (2007). Updating P300: an integrative theory of P3a and P3b. Clinical Neurophysiology : Official Journal of the International Federation of Clinical Neurophysiology, 118(10), 2128-2148.

Reed, S. A., \& Dassonville, P. (2014). Adaptation to leftward-shifting prisms enhances local processing in healthy individuals. Neuropsychologia, 56, 418-427.

Roijendijk, L., Farquhar, J., van Gerven, M., Jensen, O., \& Gielen, S. (2013). Exploring the impact of target eccentricity and task difficulty on covert visual spatial attention and its implications for brain computer interfacing. PloS One, 8(12), e80489.

Rorden, C., Karnath, H., \& Driver, J. (2001). Do neck-proprioceptive and caloric-vestibular stimulation influence covert visual attention in normals, as they influence visual neglect? Neuropsychologia, 39, 364-375.

Rossetti, Y., Rode, G., Pisella, L., Farné, A., \& Li, L. (1998). Prism adaptation to a rightward optical deviation rehabilitates lefthemispatial neglect. Nature, 395, 166-169.

Saevarsson, S., Kristjánsson, A., Bach, M., \& Heinrich, S. P. (2012). P300 in neglect. Clinical Neurophysiology, 123(3), 496-506.

Saj, A., Cojan, Y., Vocat, R., Luauté, J., \& Vuilleumier, P. (2013). Prism adaptation enhances activity of intact fronto-parietal areas in both hemispheres in neglect patients. Cortex, 49, 107-119. 
http://doi.org/10.1016/j.cortex.2011.10.009

Schindler, I., Mcintosh, R. D., Cassidy, T. P., Birchall, D., Benson, V., letswaart, M., \& Milner, A. D. (2009). The disengage deficit in hemispatial neglect is restricted to between-object shifts and is abolished by prism adaptation. Experimental Brain Research, 192, 499-510. http://doi.org/10.1007/s00221008-1585-4

Striemer, C., \& Danckert, J. (2007). Prism adaptation reduces the disengage deficit in right brain damage patients. Neuroreport, 18(1), 99-103.

Striemer, C., Sablatnig, J., \& Danckert, J. (2006). Differential influences of prism adaptation on reflexive and voluntary covert attention. Journal of the International Neuropsychological Society : JINS, 12(3), 337-349. http://doi.org/10.1017/S1355617706060553

Van der Waal, M., Farquhar, J., Fasotti, L., \& Desain, P. (2017). Preserved and attenuated electrophysiological correlates of visual spatial attention in elderly subjects. Behavioural Brain Research, 317, 415-423. http://doi.org/10.1016/j.bbr.2016.09.052

Worden, M. S., Foxe, J. J., Wang, N., \& Simpson, G. V. (2000). Anticipatory biasing of visuospatial attention indexed by retinotopically specific alpha-band electroencephalography increases over occipital cortex. The Journal of Neuroscience, 20(RC63), 1-6. 